\title{
Influencia mineragráfica en la recuperación de oro en los procesos metalúrgicos de la Minera Qero's Gold de Paucartambo- Cusco
}

\author{
Mineragraphic influence on the recovery of gold in the metallurgical \\ processes of Minera Qero's Gold de Paubustamantecartambo- Cusco
}

Alvaro Fernando Soto Mayorga ${ }^{1}$, Juan José Leguía Letellier ${ }^{2}$, Julio Danilo Bustamante Jaen ${ }^{3}$

Recibido: 01/07/2020 - Aprobado: 30/10/2020 - Publicado: 30/11/2020

\begin{abstract}
RESUMEN
Los estudios mineragráficos permitieron seleccionar el proceso metalúrgico más adecuado para una óptima recuperación de oro, cuya caracterización tanto microscópica y macroscópica, permitió experimentar pruebas metalúrgicas en moliendabilidad, cianuración en botellas, concentración centrífuga, flotación rougher. Como resultado de estas pruebas metalúrgicas en el mineral base permitió una recuperación del $94.48 \%$ en flotación selectiva con una mezcla (blending) de $36 \%$ de veta Inkaracay y $64 \%$ de veta mina Sanca 2.
\end{abstract}

Palabras claves: Mineragrafía; flotación; cianuración; oro.

\begin{abstract}
Mineragraphic studies allowed selecting the most suitable metallurgical process for optimal gold recovery, whose characterization both microscopic and macroscopic, allowed to undergo metallurgical tests on grinding, bottle cyanidation, centrifugal concentration, rougher flotation. As a result of these metallurgical tests on the base mineral, it allowed a $94.48 \%$ recovery in selective flotation with a blending of $36 \%$ Inkaracay vein and $64 \%$ Mina Sanca 2 vein.
\end{abstract}

Keywords: Mineragraphic; flotation; cyanidation; gold.

\footnotetext{
1 Universidad Nacional Mayor de San Marcos, Facultad de Ingeniería Geológica, Minería, Metalúrgica y Geográfica. Lima-Perú. Autor para correspondencia: fernando 4594@hotmail.com

2 Ingeniero Metalurgista. E-mail: juanleguia@huajayrumi.pe

3 Universidad Nacional de San Antonio Abad del Cusco, Facultad de Ingeniería Geológica, Minas y Metalúrgica. Cusco-Peru, E-mail: danilo-bi@hotmail.com
} 


\section{INTRODUCCIÓN}

En el Perú existen depósitos orogénicos, los que son explotados de manera informal y poco organizada. Qero's Gold se sitúa en la provincia de Paucartambo en la región del Cusco, forma parte de la Cordillera Oriental en cuya geología se originan yacimientos cordilleranos, también conocido como filones de cuarzo, vetas en zonas de cizalla, yacimientos mesotermales, yacimientos orogénicos, los cuales tienen como peculiaridad principal presentar poco volumen y una ley alta (hasta $\mathrm{kg} / \mathrm{T}$ ) (Acosta, 2012).

Diversos estudios evidencian la utilidad de la aplicación de los estudios mineragráficos dentro de las operaciones unitarias en metalurgia y se plantea etapas de preconcentración para optimizar la recuperación de oro diseminado con presencia de cuarzo y la flotación selectiva de oro en menas auríferas (Audebaud, 1973), (Quispe, 2018).

El presente trabajo buscó un proceso metalúrgico adecuado para mejorar la recuperación del oro en base a la mineragrafía. Para lo cual se realizó una caracterización mineralógica y pruebas metalúrgicas de moliendabilidad, concentración gravimétrica, flotación rougher y lixiviación por agitación en botellas (Azareño, 2009).

\section{MATERIALES Y MÉTODOS}

El mineral del yacimiento de oro de Qero's Gold, inicialmente se sometió a un muestreo no probabilístico de tipo intencionado, seleccionando ocho kilos de muestra para análisis químico de cabeza, concentrado y relave. Siete kilos de muestras de mena y concentrado para análisis mineragráficos microscópico y macroscópico; para el estudio de textura, asociaciones mineralógicas, tamaño de grano, etc. Trecientos kilos de muestra para pruebas metalúrgicas a nivel de laboratorio tanto en mineral como concentrado.

\subsection{Materiales y equipos}

- Chancadora de mandíbulas.

- Molino de bolas.

- Celda de flotación.

- Concentrador centrifugo Falco F-140.

- Columnas de lixiviación.

- Microscopio electrónico de barrido.

- Microscopio de luz polarizada.

- Difractómetro.

\subsection{Caracterización mineragráfica}

Sirve para el análisis de minerales opacos utilizando un microscopio polarizante de reflexión o luz reflejada o luz incidente a diferencia de la mineralogía óptica donde se utiliza el microscopio de luz transmitida y para el trabajo de investigación.
Al caracterizar mineragráficamente cada muestra, se identifican las especies mineralógicas y se determina el grado de liberación de cada especie. Toda esta información ayudará a obtener información aplicable a la optimización de las pruebas metalúrgicas

Actualmente, la principal herramienta para el análisis de la superficie radicular es el uso de la microscopia electrónica de barrido (MEB), es un instrumento que permite la observación y análisis de las superficies de materiales orgánicos e inorgánicos (Estrada et al., 2009). Así mismo se aplicó la microscopia electrónica de barrido para la identificación y verificación de la presencia de oro en solución sólida con los sulfuros o minerales submicroscópicos de oro, debido a que la recuperación metalúrgica es muy variable y no se puede percibir a simple vista ni con el microscopio de luz polarizada, que minerales están perjudicando la recuperación en el concentrador centrifugo, tampoco se puede determinar el porcentaje de oro libre presente en el concentrado y mucho menos la cantidad de mineral de oro que queda atrapado en la matriz de cuarzo-pirita.

Se usa las siguientes abreviaciones al observar en el microscopio: cuarzo (Qz), pirita (py), goethita (goe), gangas (GGs), oro (Au), arsenopirita (aspy), sulfosal (sul), calcopirita (cpy), hematita (he), electrum (el) y arsenopirita (apy). Para las pruebas metalúrgicas malla (m)

\subsection{Pruebas metalúrgicas}

Se efectuó una preparación mecánica de las muestras para obtener $100 \%$ - $10 \mathrm{~m}$ Tyler, para posteriormente obtener una muestra representativa mediante homogenizado y cuarteo, posteriormente se formaron los compósitos de trabajo a los que etiquetamos como muestra 1,2 y 3 . Se realizó una caracterización de la cabeza mediante análisis químico, se prosiguió con las pruebas de moliendabilidad en un molino de bolas estándar de laboratorio de 8"x 10" a un porcentaje de sólidos de $66 \%$. Las pruebas de concentración gravimétrica se realizaron mediante el equipo Falcón L-40 a una fuerza de gravedad de $98 \mathrm{G}$ y una presión de agua de 1.5 PSI, adicionalmente se utilizó $10 \mathrm{~kg}$ de muestra con granulometría de $65 \%-200 \mathrm{~m}$ y un flujo de alimentación de $1800 \mathrm{ml} / \mathrm{min}$. La lixiviación por agitación en botellas se realiza a un $\mathrm{pH}$ entre $10.5-11.0$, con $30 \%$ de sólidos, $2 \mathrm{~kg}$ de mineral con $85 \%$-m200, la fuerza de cianuro es 1500 $\mathrm{ppm}$. La flotación rougher a $32 \%$ de sólidos, con mineral a un tamaño de $65 \%-200 \mathrm{~m}$ y $85 \%-\mathrm{m} 200$, los reactivos empleados en las pruebas de flotación fueron el $\mathrm{CuSO}_{4}$ como agente activador de los sulfuros presentes, el A-404 como colector especifico de las especies portadoras de $\mathrm{Au}$, Z-11 como colector secundario y MIBC como espumante.

\section{RESULTADOS Y DISCUSIÓN}

\subsection{Mineralización}

Se presenta la veta Inkaracay con un afloramiento aproximado de $130 \mathrm{~m}$, la cual tiene una potencia que varía entre 15 y $20 \mathrm{~cm}$, cuya profundidad de la veta llega a los $60 \mathrm{~cm}$, el ensamble es Qz-Py-Ca-Au se observa carbonatos (ankerita) así como oro grueso y fino en las microfracturas de la pirita, $\mathrm{y}$ oro en forma diseminada en el cuarzo lechoso con una ley promedio de promedio $20 \mathrm{~g} / \mathrm{T}$. 
La veta Mina Sanca 2 presenta un afloramiento de más de $350 \mathrm{~m}$, con una potencia en promedio de $17 \mathrm{~cm}$, el ensamble es Qz-Py-Ca-Au, se aprecia oro grueso en forma de oro nativo entrampado dentro en una matriz de cuarzo gris (Figura 1), también se presenta como laminas en el contacto cuarzo y pizarra, el oro fino se muestra como relleno en las micro fracturas de la pirita, mientras que el oro fino diseminado en el cuarzo, presenta una ley en promedio de $45 \mathrm{~g} / \mathrm{T}$.

La zona de stockwork corresponde a una estructura mineralizada con presencia de varias familias de vetillas de cuarzo lechoso; las venillas predominantes tienen una dirección SE-NO, el ensamble en la roca es de tipo Qz-Py-Apy-Au-Ag presenta alta piritización, el mineral encapsulado esta entre cuarzo y pirita, algunas venillas superan los $250 \mathrm{~g} / \mathrm{T}$ obteniendo así una explotación en global un área de $1,000 \mathrm{~m}^{2}$ aproximadamente con una ley de $8 \mathrm{~g} / \mathrm{T}$ teniendo una mineralización probada de 40,000 T a $15 \mathrm{~m}$ de profundidad y mineralización probable $120 \mathrm{~m}$ en profundidad.

\subsection{Análisis mineralógico de grados de liberación \\ 3.2.1 Veta Inkaracay}

Las microfotografías de la Figura 2a muestran partículas libres de arsenopirita (apy) al $89.17 \%$ en grado de liberación, así como grano mixto binario de oro nativo (Au), Pirita (Py), Goetita (Goe) y ganga (GGs). Así como presencia de calcopirita $(\mathrm{Cp})$ incrustada en la ganga.

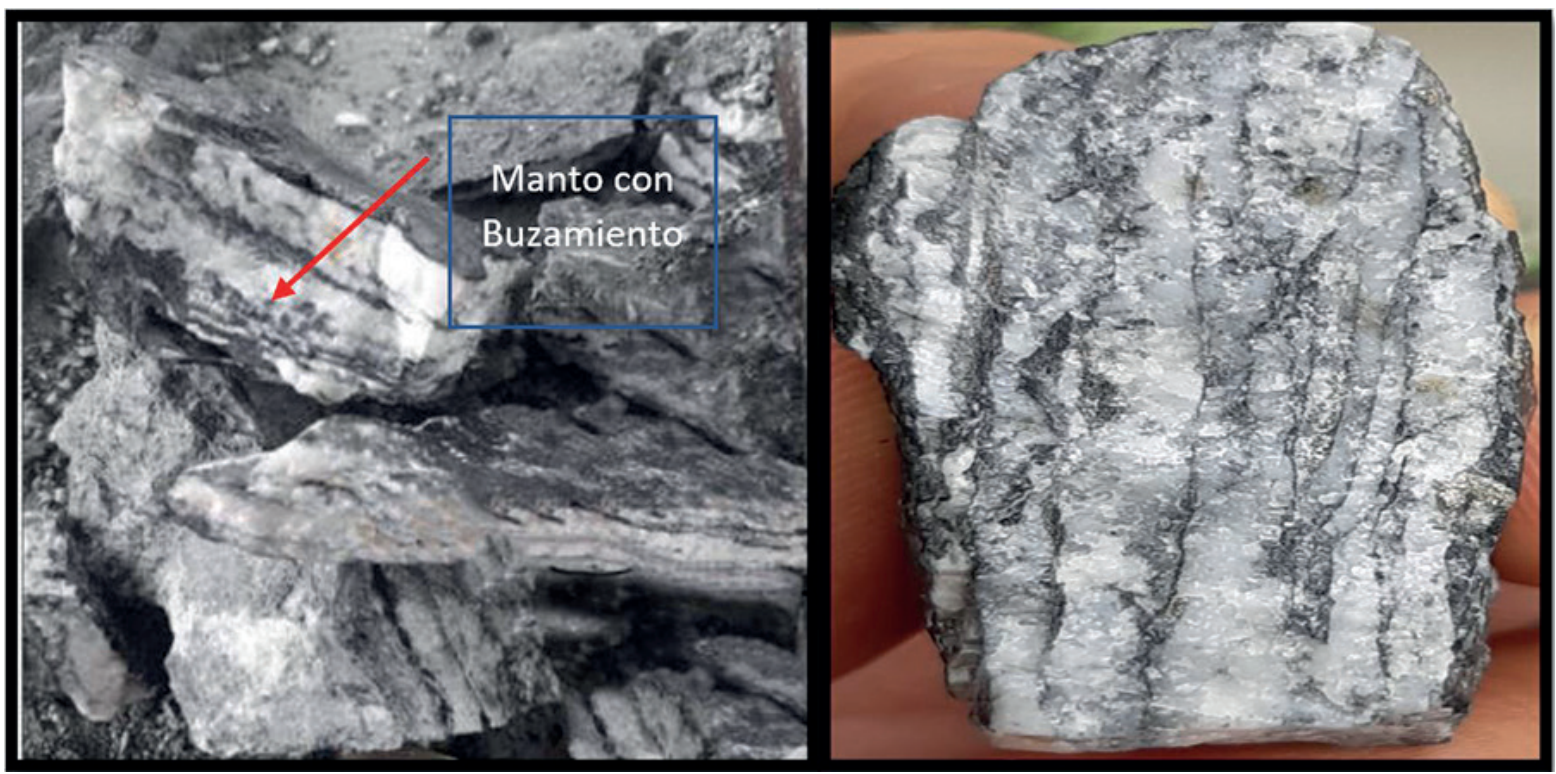

Figura 1. Zona con diseminación de Oro en venillas en diversas direcciones

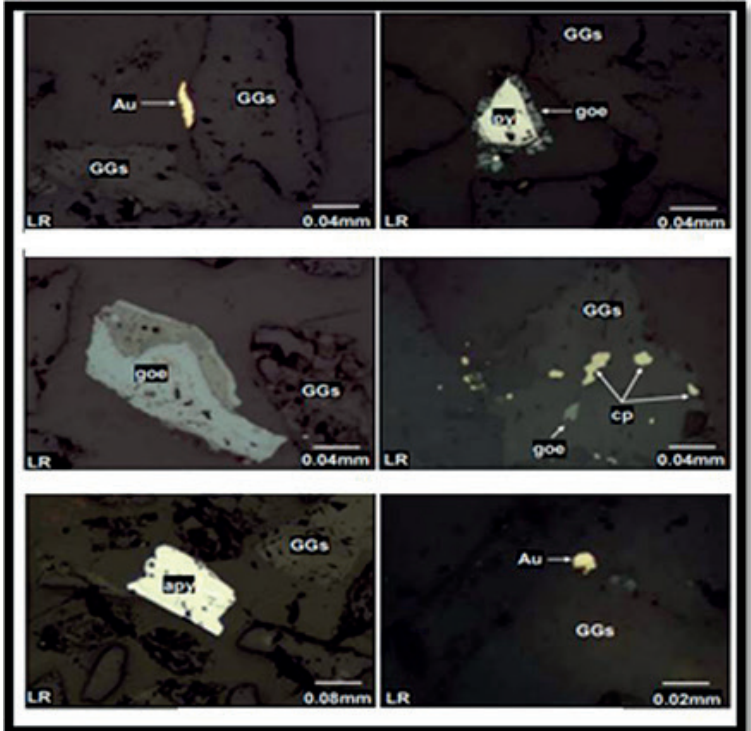

(a)

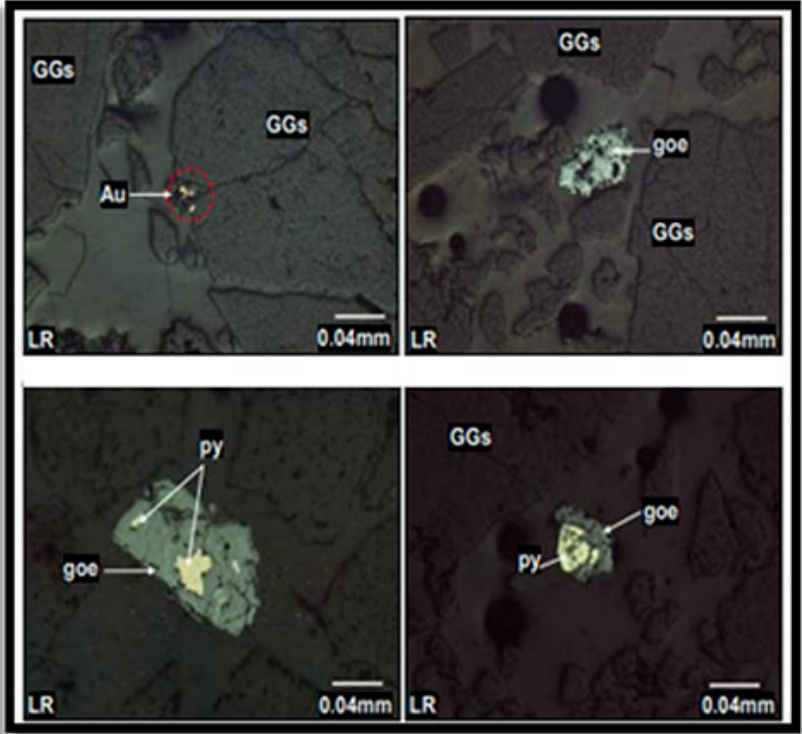

(b)

Figura 2. Microfotografía grado de liberación Veta Inkaracay a malla +100 
Por otro lado, en la Figura $2 \mathrm{~b}$ presentan grano mixto binario de gangas no metálicas y oro nativo (GGs-Au). Al igual que granos libres de minerales de gangas no metálicos (GGs) y goethita (Goe). Como también granos mixtos binarios de pirita y goethita.

\subsubsection{Veta Mina Sanca 2}

De la misma manera se realizó un análisis mineralógico para la Veta Mina Sanca 2, se puede deducir que en la microfotografía de la figura 3, se aprecia trazas de oro con un grado de liberación de oro mínimo $76.49 \%$ no tiene una buena separación con referencia a sus gangas $99.91 \%$ podemos ver uniones con goetitas al $93.76 \%$ en grado de liberación.

También se puede apreciar que cuanto más molido este el mineral tendrá un mayor grado de liberación esto a una malla -200 en la microfotografía se ve al oro separado de sus gangas.

\subsection{Análisis por difracción de Rayos $X$}

La difracción de rayos $\mathrm{X}$ realizados a las muestras de las vetas Inkaraccay y Mina Sanca, se observa la presencia de cuarzo, muscovita, dolomita, pirita, albita, apatito, cuarzo, clinocloro y rutilo (Figura 4 y 5 ).

\subsection{Análisis por microscopia electrónica de barrido}

Se estudia al concentrado de oro gravimétrico a fin de identificar presencia de oro en solución sólida con los sulfuros o minerales submicroscópicos de oro.

En la Figura 6a se observa aproximadamente un $20 \%$ de presencia de pirita, trazas de electrum mineral de oro y

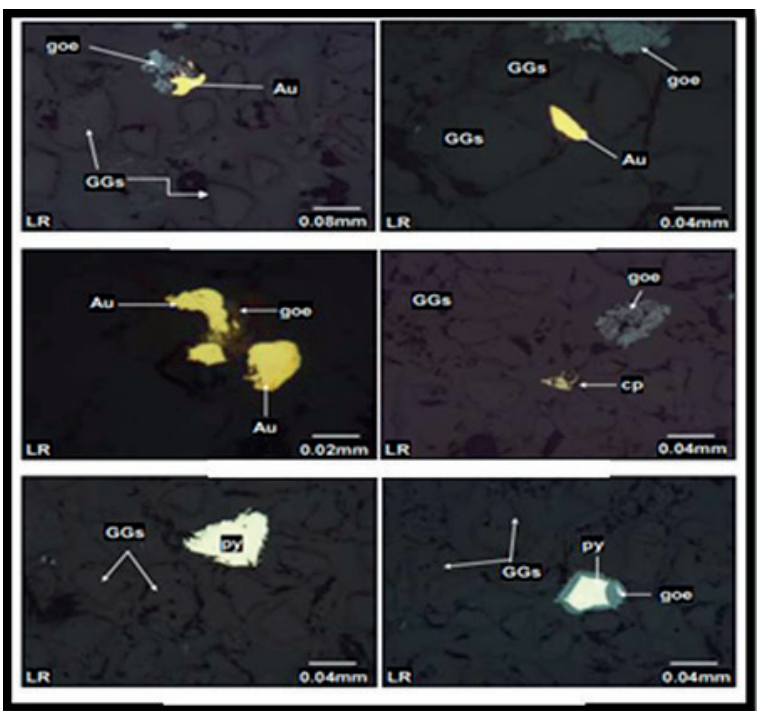

Figura 3. Microfotografía grado de liberación Veta Mina Sanca 2 malla -200

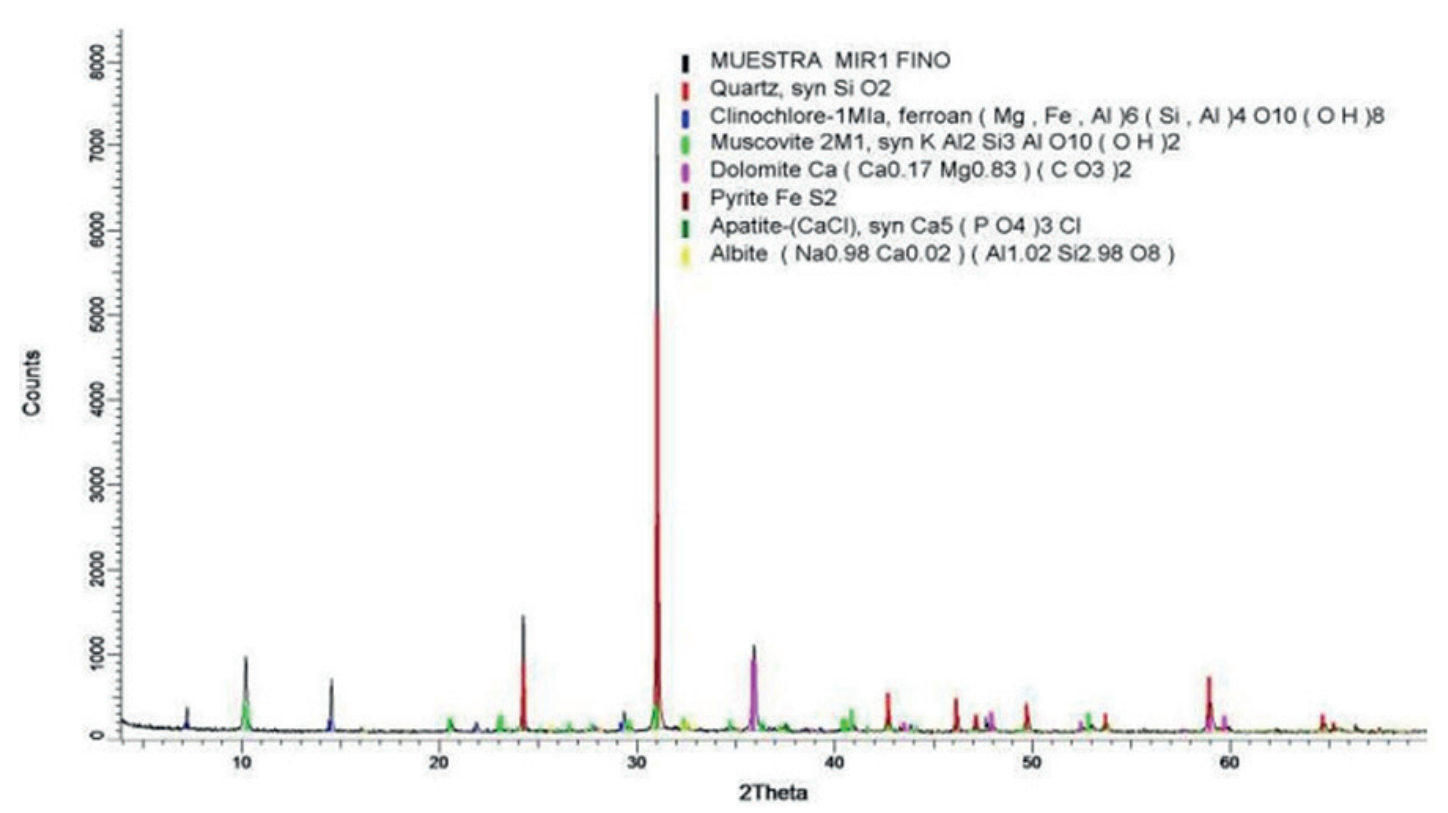

Figura 4. Difractograma de la Muestra MIR 1 Veta Inkaracay 
plata, así como presencia de arsenopirita en el concentrado gravimétrico, la parte grisácea corresponde a granos de cuarzo con tamaños menores a $300 \mu \mathrm{m}$.

En la Figura $6 \mathrm{~b}$ al aplicar un zoom en el microscopio electrónico de barrido al oro podemos ver inclusiones de pirita lo cual conlleva a un encapsulamiento de pirita dentro de una matriz de cuarzo; el mineral de oro presente como electrum forma el $93 \%$ de oro y $7 \%$ de plata.

\subsection{Composición mineralógica del concentrado}

Mediante el uso del microscopio electrónico de barrido, se determina la composición porcentual de los minerales presentes en la muestra (Tabla 1), donde la especie de interés de estudio es el oro y representa el $0.1 \%$ en volumen de la muestra en trazas de electrum.
Tabla 1. Composición Mineralógica Porcentual

\begin{tabular}{|c|c|}
\hline Minerales & Porcentaje \\
\hline Cuarzo & $63 \%$ \\
\hline Pirita & $18 \%$ \\
\hline Calcita & $6 \%$ \\
\hline Arsenopirita & $5 \%$ \\
\hline Hematita & $4 \%$ \\
\hline \multicolumn{2}{|c|}{ Especies menores } \\
\hline Alumina & $2 \%$ \\
\hline Sulfosal & $1.6 \%$ \\
\hline esfalerita & $0.1 \%$ \\
\hline electrum & $0.1 \%$ \\
\hline
\end{tabular}

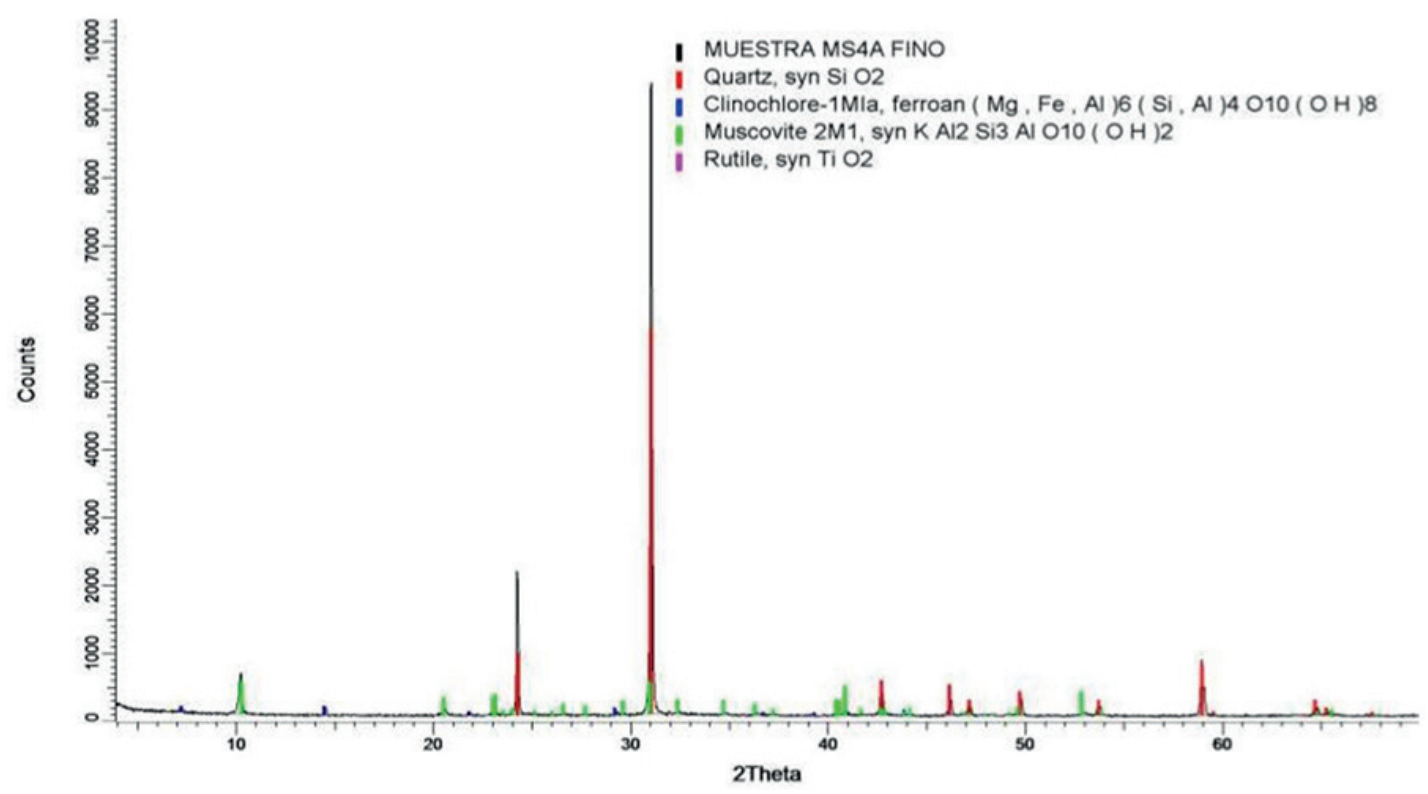

Figura 5. Difractograma de la Muestra MS 4 Veta Mina Sanca 2
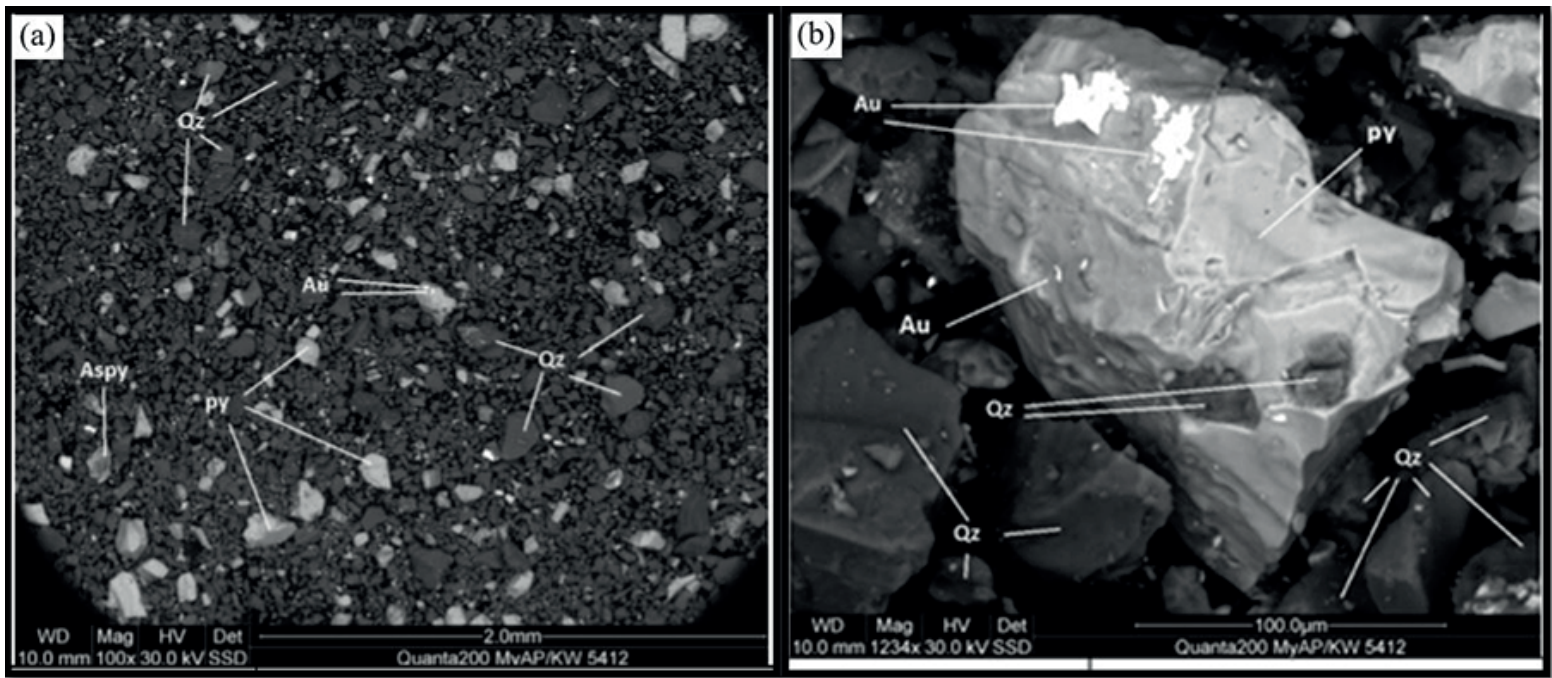

Figura 6. Muestra un grano de pirita a $150 \mu \mathrm{m}$ con inclusiones de $20 \mu \mathrm{m}$ y $2 \mu \mathrm{m}$ de oro en forma de electrum así como inclusiones de cuarzo 


\subsection{Análisis metalúrgico}

3.6.1 Porcentaje de mezcla o blending mineralógico Esta tabla 2 muestra el porcentaje respectivo de mezcla (blending), que están codificadas como muestra 1, 2 y 3, utilizados para el análisis de moliendabilidad, concentración gravimétrica, flotación rougher y lixiviación directa.

Tabla 2. Codificación, peso de las muestras para los respectivos procesos metalúrgicos y porcentaje de mezcla o blending

\begin{tabular}{llcc}
\hline & Código & Peso (kg) & Porcentaje (\%) \\
\hline \multirow{2}{*}{ Muestra - 1 } & V. Inkaracay & 28 & $63.64 \%$ \\
& V. Mina San. 2 & 16 & $36.36 \%$ \\
\hline \multirow{2}{*}{ Muestra - 2 } & V. Inkaracay & 18 & $36.00 \%$ \\
& V. Mina San. 2 & 32 & $64.00 \%$ \\
\hline \multirow{2}{*}{ Muestra - 3 } & V. Inkaracay & 28 & $49.12 \%$ \\
& V. Mina San. 2 & 29 & $50.88 \%$ \\
\hline
\end{tabular}

En la tabla 3 se observa el análisis químico de cabeza del mineral para el respectivo balance metalúrgico.

Tabla 3. Análisis químico de cabeza

\begin{tabular}{lcccc}
\hline & \multicolumn{4}{c}{ Leyes } \\
Código de muestras & $\mathrm{Au}$ & $\mathrm{Ag}$ & $\mathrm{Fe}$ & $\mathrm{As}$ \\
& $(\mathrm{g} / \mathrm{T})$ & $(\mathrm{g} / \mathrm{T})$ & $(\%)$ & $(\%)$ \\
\hline Muestra -1 & 0.16 & $<0.5$ & 4.15 & 0.15 \\
Muestra -2 & 2.92 & $<0.5$ & 3.47 & 0.06 \\
Muestra -3 & 1.95 & $<0.5$ & 5.06 & 0.12 \\
\hline
\end{tabular}

\subsubsection{Moliendabilidad}

Los resultados de las pruebas de moliendabilidad, donde a mayor tiempo de residencia en el molino se logra un mayor grado de liberación, y se obtiene una liberación de $65 \%$ -200 malla en un tiempo promedio de 19.37 minutos con una granulometría optima de 100 micrones.
Tabla 4. Resultados de la prueba de moliendabilidad

\begin{tabular}{lccc}
\hline \multirow{2}{*}{ Código de Muestras } & \multicolumn{3}{c}{ Resumen Tiempo Molienda } \\
& $\%$-m200 & Tiempo $(\mathrm{min})$ & P80 $(\boldsymbol{\mu m})$ \\
\hline Muestra -1 & 65 & 16.40 & 110 \\
& 85 & 29.50 & 65 \\
\hline Muestra -2 & 65 & 31.20 & 100 \\
& 85 & 43.60 & 68 \\
\hline Muestra -3 & 65 & 17.50 & 115 \\
& 85 & 27.60 & 67 \\
\hline
\end{tabular}

\subsubsection{Concentración Gravimétrica}

Se aplica la concentración gravimétrica ya que es un método adecuado para separar y concentrar oro grueso libre, también por su alto peso específico. Se realizaron dos pruebas de concentración gravimétrica en Falcon L-40, con las muestras denominadas Muestra -2 y Muestra -3 .

En la tabla 5 se observa un bajo porcentaje de recuperación por el método de concentración gravimétrica solo recuperando oro grueso a medio y no el oro fino.

Tabla 5. Concentración Gravimétrica

\begin{tabular}{lcccc}
\hline \multirow{2}{*}{ Muestra } & \multicolumn{2}{c}{ Ley $(\mathrm{g} / \mathrm{t})$} & \multicolumn{2}{c}{ Recuperación (\%) } \\
& $\mathrm{Au}$ & $\mathrm{Ag}$ & $\mathrm{Au}$ & $\mathrm{Ag}$ \\
\hline Conc. Grav. - Muestra -2 & 89.78 & 7 & 49.19 & 13.40 \\
Conc. Grav. - Muestra -3 & 132 & 7.7 & 58.61 & 14.63 \\
\hline
\end{tabular}

\subsubsection{Lixiviación por agitación en botellas}

La Figura 7 muestra el porcentaje de recuperación de oro y plata con respecto a un tiempo de 72 horas, donde la baja recuperación de oro y plata (menor que 19\%) está relacionado al efecto preg-robbing, este efecto ocurre en presencia de carbón, la muestra utilizada presenta un contenido de $1.12 \%$ de carbón, además es de tipo refractario al proceso de lixiviación debido a que el oro se encuentra

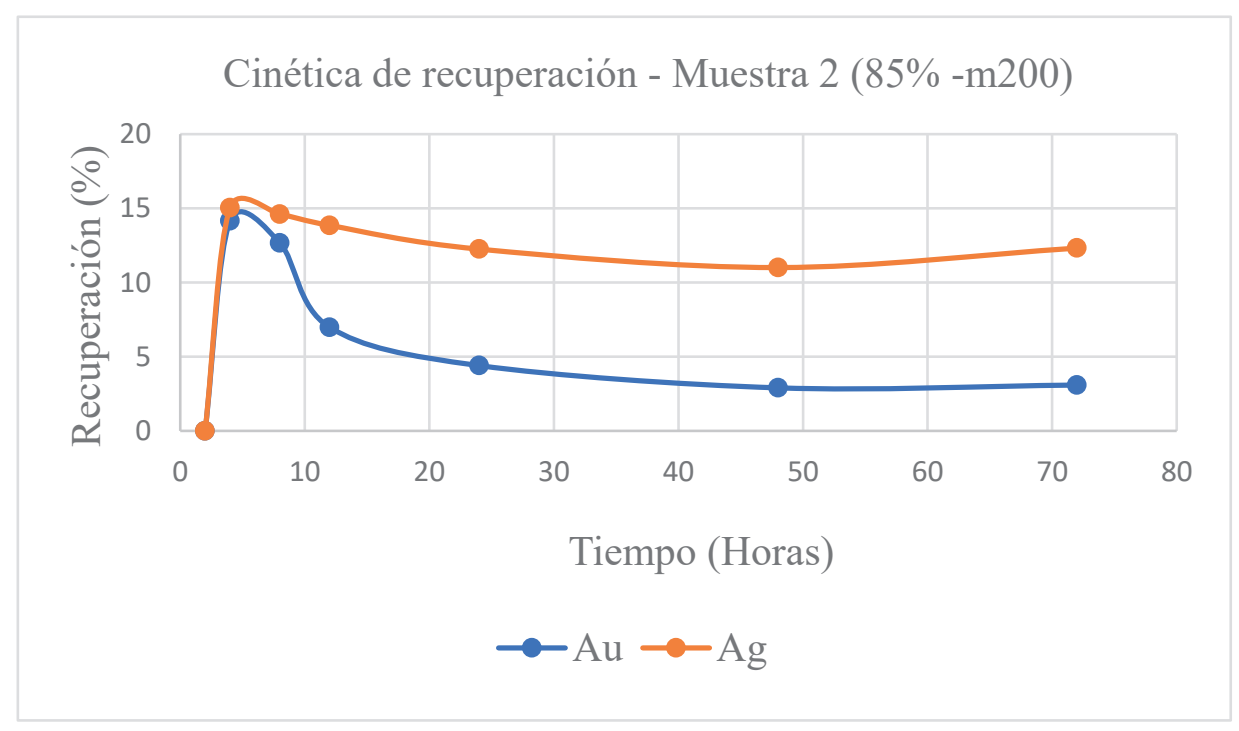

Figura 7. Cinética de recuperación - Muestra 2 (85\% -m200) 
ocluido en una matriz de pirita y presenta contenidos de arsenopirita, este tipo de contenidos con lleva a un consumo del cianuro que es $2 \mathrm{Kg} / \mathrm{T}$ a las 72 horas como efecto adverso la recuperación de oro. (Figura 8).

\subsubsection{Flotación rougher}

En la tabla 6 para llegar una recuperación del 95.54\% de oro se debe trabajar con una granulometría de $85 \%$ $200 \mathrm{~m}$ a un $\mathrm{pH}$ de 7.7 para evitar la flotación de pirita en lugar del oro, como reactivos para la flotación se utilizó al CuSO4 para deprimir la pirita, colector primario al A-404 y colector secundario Z-11, así como espumante al MIBC

\section{DISCUSION}

Con el uso de la mineragrafía para tener una caracterización del mineral más profundo y fidedigno, se derivó a ejecutar un análisis microscópico tanto de luz polarizada como la microscopia electrónica de barrido, con lo cual se pudo observar en las asociaciones mineralógicas un encapsulamiento de oro en una matriz de cuarzo y pirita, presencia de material carbonáceos, sulfosales de cobre y plomo las cuales impiden que la liberación del material valioso sea buena, parte de las gangas que son perjudiciales en el proceso metalúrgico son las arsenopiritas que actúan como cianicidas al igual que el manto de 107 piedra pizarra que cubre la matriz de cuarzo gris y que por su dureza impide que la liberación del metal valioso en molienda no sea la más adecuada.

Se determinó que el proceso metalúrgico más adecuado para el tipo de mineral trabajado es la flotación selectiva con una mezcla (blending) de 36\% mineral de veta Inkaracay y $64 \%$ de mineral veta Mina Sanca 2, a una granulometría de $100 \mu \mathrm{m}$, un porcentaje de solidos de $34 \%$ y a un $\mathrm{pH}$ ligeramente alcalino de 7.9 , se brinda las condiciones de acondicionamiento necesario para obtener un concentrado de oro al $95.54 \%$ en recuperación.

\section{CONCLUSIONES}

- Según la mineragrafía con el uso del microscopio de luz polarizada, el oro se encuentra entre los tamaños de 10 a 60 micras con un grado de liberación del $80 \%$; y entre los tamaños de 20 a 30 micras el oro se encuentra en intercrecimiento con el cuarzo, goethita, pirita y arsenopirita.

- Al caracterizar el mineral con el uso de un microscopio electrónico de barrido (MEB), se demuestra que la composición del electrum es de $92.99 \%$ de $\mathrm{Au}$ y $7.01 \%$ de Ag, la Pirita y la Arsenopirita contienen oro en su red cristalina con tamaños de oro de hasta 2 micras

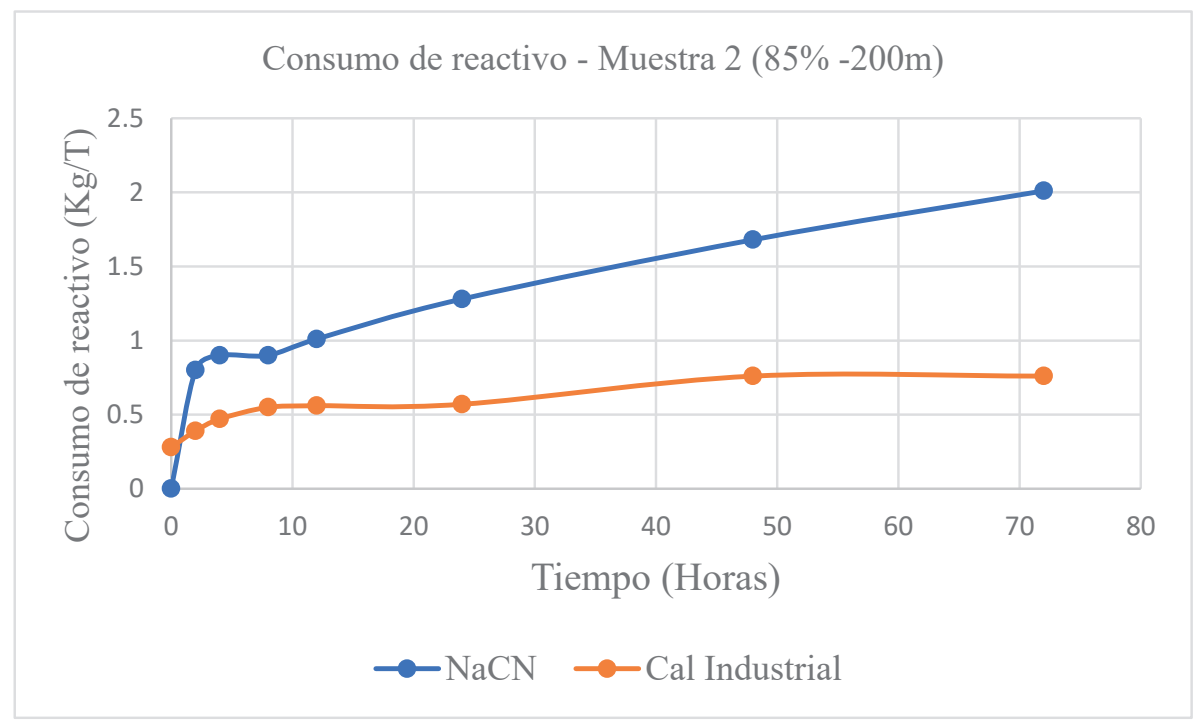

Figura 8. Consumo de reactivo - Muestra 2 (85\% -200m).

Tabla 6. Balance metalúrgico flotación Ro - Muestra- 2 (85\% -m200)

\begin{tabular}{|c|c|c|c|c|c|c|c|c|c|}
\hline \multirow{3}{*}{ Productos } & \multirow{3}{*}{$\begin{array}{l}\text { Peso } \\
(\%)\end{array}$} & \multicolumn{4}{|c|}{ Ley } & \multirow{2}{*}{\multicolumn{4}{|c|}{ Recuperaciones (\%) }} \\
\hline & & \multicolumn{2}{|c|}{$(g / t)$} & \multicolumn{2}{|c|}{$(\%)$} & & & & \\
\hline & & $\mathrm{Au}$ & $\mathrm{Ag}$ & As & $\mathrm{Fe}$ & $\mathrm{Au}$ & $\mathrm{Ag}$ & As & $\mathrm{Fe}$ \\
\hline Conc. Ro. (P01) & 5.14 & 57.30 & 9.50 & 0.87 & 26.98 & 95.54 & 50.74 & 88.75 & 39.23 \\
\hline Relave & 94.86 & 0.15 & $<0.50$ & 0.01 & 2.27 & 4.46 & 49.26 & 11.25 & 60.77 \\
\hline Cab. Calculada & 100.00 & 3.08 & 0.96 & 0.05 & 3.54 & 100.00 & 100.00 & 100.00 & 100.00 \\
\hline Cab. Ensayada & & 2.91 & $<0.50$ & 0.06 & 3.47 & & & & \\
\hline
\end{tabular}


- Se determinó que el mejor procedimiento metalúrgico para obtener alta recuperación de oro es la flotación selectiva debido a sus características y condiciones del mineral; se puede concluir también que la granulometría repercute de manera directa debido a que se tuvo mejor porcentaje de recuperación a una granulometría más fina $85 \%-200 \mathrm{~m}$ a diferencia de una granulometría de $65 \%-200 \mathrm{~m}$. logrando un $95.54 \%$ de recuperación que implica una mejora significativa en el proceso.

\section{REFERENCIAS}

Acosta, H. et. A. (2012). Recuperación de oro y plata de minerales sulfurados en una matriz de cuarzo. Revista Del Instituto de Investigación de La Facultad de Ingeniería Geológica, Minera, Metalurgica y Geográfica, 10(19), 28-37. https:// revistasinvestigacion.unmsm.edu.pe/index.php/iigeo/ article/view/503

Audebaud, E. (1973). Geología de los cuadrángulos de Ocongate y Sicuani, hojas 28-t y 29-t. Repositorio Institucional INGEMMET, 72. https://repositorio.ingemmet.gob.pe/ handle/20.500.12544/143
Azareño, A. et. A. (2009). Factores que afectan la selección del proceso metalúrgico para beneficiar minerales complejos de oro. Revista Del Instituto de Investigación de La Facultad de Ingeniería Geológica, Minera, Metalurgica y Geográfica, 12(24), 49-55. https://revistasinvestigacion.unmsm.edu.pe/ index.php/iigeo/article/view/356

Estrada, W., Rodriguez, J., \& Solís, J. (2009). Recubrimientos delgados obtenidos por procedimientos físico-químicos. In Universidad Nacional de Ingeniería. Editorial Universitaria. https://docplayer.es/170554751-Recubrimientos-delgadosobtenidos-por-procedimientos-fisico-quimicos-primeraedicion-digital-enero-lima-peru.html

Quispe, V. (2018). Estudio mineragráfico y determinación microscópica del grado de liberación de los minerales sulfurados de cobre. In Universidad Nacional de San Agustin de Arequipa (Issue Ley 29497). http://repositorio. unsa.edu.pe/handle/UNSA/6724 